\title{
Decisiones, bienestar y desarrollo humano en departamentos de bienestar estudiantil. Discusión de hallazgos
}

Isabel Cristina Lopera. Universidad de Salamanca Jonathan Echeverri Álvarez. Universidad EAFIT

\section{Resumen}

El presente estudio cualitativo tuvo como objetivo explorar la arquitectura para la toma de decisiones que se promueve en los programas institucionales de bienestar y desarrollo humano, ofrecidos en tres instituciones de educación superior de la ciudad de Medellín y el municipio de Bello en Antioquia, Colombia. Para lograrlo, se conocieron los programas institucionales y se detectaron los componentes asociados con la arquitectura para la toma de decisiones. Se realizaron quince entrevistas semiestructuradas a líderes encargados de los programas institucionales en las universidades objeto de estudio y un grupo focal con estudiantes universitarios. Para el análisis se utilizó un proceso de codificación y triangulación de las entrevistas. Esta información fue contrastada con la revisión documental y la investigación empírica, las cuales enfatizan la importancia de diseñar contextos para aumentar el bienestar de las personas. Finalmente, sugerimos un programa de formación en toma de decisiones para los arquitectos encargados de estas áreas.

Palabras clave: toma de decisiones; bienestar; desarrollo humano; nudge; formación.

\section{Resumo}

Decisões, bem-estar e desenvolvimento humano em departamento de bem-estar estudantil. Discussão de resultados. Este estudo qualitativo teve como objetivo explorar a arquitetura para a tomada de decisão promovida nos programas institucionais de bemestar e desenvolvimento humano, oferecidos em três instituições de ensino superior na cidade de Medellín e no município de Bello, em Antioquia, na Colômbia. Para realizá-lo, foram analisados os programas institucionais e detectados os componentes associados à arquitetura para tomada de decisão. Foram realizado quinze entrevistas semi-estruturadas aos líderes responsáveis dos programas institucionais nas universidades em estudo. Para a análise, foi utilizado um processo de codificação e triangulação das entrevistas. Esta informação foi contrastada com a revisão documental e a pesquisa empírica, que enfatizam a importância de projetar contextos para aumentar o bem-estar das pessoas. Finalmente, sugerimos um programa de Formação na tomada de decisão para os arquitetos responsáveis destas áreas.

Palavras-chave: decisões; bem-estar; desenvolvimento humano; empurrão; treinamento.

\section{Summary}

Decisions, well-being and human development in departments of student well-being. Discussion of findings. The objective of this qualitative study was to explore the architecture for decision making promoted in the institutional programs of welfare and human development, offered in three institutions of higher education in the city of Medellin and the municipality of Bello in Antioquia, Colombia. To achieve this, we got the information about the institutional programs. Then, we extract the components associated with the architecture for decision making. Fifteen semi-structured interviews were carried out with leaders in charge of institutional programs at the universities under study. For the analysis, we used a process of coding and triangulation of the interviews. This information was contrasted with the documentary review and empirical research, which emphasize the importance of designing contexts to increase human well-being. Finally, we suggest a training program in decision making for the architects in charge of these areas.

Keywords: decision-making; welfare; human development; nudge; Training. 
Los departamentos de bienestar estudiantil son áreas dentro de las universidades que ofrecen programas para complementar la formación académica e impactar el bienestar de la comunidad. Su marco de acción es delimitado a la vida universitaria, pero puede incluir actividades materiales, culturales y espirituales extra-curriculares (Contecha, 2008, Valarezo y Túñez, 2014). Debido a la multiplicidad de factores que se relacionan con el sentido de "estar bien", su intervención requiere un amplio panorama de posibilidades (Kitayama, Markus, \& Kurokawa, 2000). El concepto de bienestar incluye indicadores objetivos de buena salud, seguridad financiera y conceptos asociados con eudaimonia, es decir, el significado y el propósito de la vida. También integra el bienestar subjetivo, producto de la evaluación del componente cognitivo de las experiencias afectivas (cfr. Diener, Suh, Lucas, \& Smith, 1999; Veenhoven, 2009) y circunscribe el término felicidad para referirse a cómo las personas expresan y comunican sentimientos o condiciones favorables. Por lo tanto, implica tres componentes: "1. Afecto, ¿felicidad ahora?, ¿felicidad en general?; 2. Cognición, ¿satisfacción ahora? ¿Satisfacción en general?; 3. Eudaimonía, ¿experiencia significativa ahora? ¿Significativa en general?" (Oishi, 2010, p. 37) (Traducción nuestra) ${ }^{1}$.

La psicología positiva propone que la felicidad de un sujeto se mueve respecto a la siguiente ecuación: Felicidad = Línea de base biológica + Condiciones de tu vida + Actividades voluntarias (Haidt, 2006, p. 117; crf. Lyubomirsky, Sheldon, \& Schkade 2005; Seligman, 2002). La línea de base biológica representa una constitución que se despliega en el ciclo vital del ser humano con implicaciones en el temperamento, la personalidad, el tono emocional, entre otras. Las condiciones de vida incluyen hechos que no se pueden modificar, como raza, sexo, edad o discapacidad, y también aspectos que sí se pueden modificar, como riqueza, estado civil o lugar de residencia. Estas condiciones son constantes a lo largo del tiempo, o al menos durante un periodo de vida, y son el tipo de circunstancias a las que probablemente se adapten las personas. Las actividades voluntarias por otro lado, son las cosas que las personas eligen hacer, como la meditación, el ejercicio, aprender nuevas habilidades o tomarse unas vacaciones.

Al respecto, y teniendo en cuenta la importancia de las actividades voluntarias, en Colombia, la Asociación Nacional de Universidades -ASCUN- estableció tres áreas temáticas para el bienestar universitario: deporte y actividad física; cultura; desarrollo humano y promoción de la salud (ASCUN, 2017). El propósito común es la formación integral de los estudiantes en la educación superior (International Association of Universities, 2016). Sin embargo, la implementación de los programas supone una serie de desafíos institucionales para cumplir con éxito su misión. Decisiones centralizadas, falta de formación de los encargados, desconocimiento de la población atendida y pobre participación de los estudiantes, pueden resultar en factores restrictivos que disminuyen el impacto de su gestión. Incluso, se presentan prácticas que oscilan desde un completo asistencialismo (subsidios económicos), a uno basado en el desarrollo humano (construcción de capacidades) (Contecha \& Jaramillo, 2011; Montoya-Vásquez, UrregoVelásquez, \& Páez-Zapata, 2014).

El enfoque del desarrollo humano surge de la necesidad ética de no separar el proceso del crecimiento económico y su impacto en el bienestar de las personas (Alkire, 2002; Nussbaum, 2012; Sen, 1996). Las publicaciones de Thaler y Sunstein (2011) y el Grupo Banco Mundial (2015) sugieren la importancia de diseñar entornos que favorezcan la toma de decisiones para el bienestar y el desarrollo humano. Desde un contexto organizacional específico hasta el diseño e implementación de una política pública.

Thaler y Sunstein (2009) proponen la idea que todo grupo u organización disponen de un contexto inconsciente o deliberado para la toma de decisiones. Es decir, en ocasiones un grupo u organización propone un plan que intenta encausar las decisiones de los individuos que la componen y en otros escenarios se tejen estructuras de elección que no son deliberados o planeados pero que igualmente tienen una incidencia notable en las decisiones de las personas. A este contexto lo denominan "arquitectura para la toma de decisiones". Y el "arquitecto(s) de las decisiones" es aquel que tiene la responsabilidad de organizar el contexto en el que se toman las decisiones. Los autores proponen el concepto de nudge, palabra que en el idioma inglés significa "Empujar suavemente o dar un golpecito en las costillas, sobre todo con el codo a fin de avisar, recordar o amonestar suavemente a otro" (p. 13). El nudge en el ámbito de la elección implica estimular, incentivar o encaminar en la toma de decisiones.

Estas ideas invitan a pensar que el bienestar y el desarrollo humano se ve influenciado por las decisiones que toman los individuos y también por las oportunidades de elección que se ofrecen en los contextos organizacionales, a través por ejemplo de un programa 
institucional de bienestar estudiantil (Kahneman, 2012; Nussbaum, 2012; Saldarriaga, 2010; Sen, 1996; Thaler \& Sunstein, 2009; World Bank Group, 2015).

Entre las investigaciones empíricas más relevantes, se encuentran las publicaciones de Armitage, Bene, Charles, Johnson y Allison, (2012); Helliwell, BarringtonLeigh, Harris y Huang, (2010); Oishi (2010). Estas investigaciones exponen la sensibilidad del bienestar de los seres humanos ante pequeñas modificaciones de contexto; también resaltan, por ejemplo, la importancia de poder decidir, sin obligaciones arbitrarias externas, en cada estadio del ciclo vital. En otras palabras, a mayores oportunidades y formación de autonomía, que potencia la ejecución de actividades voluntarias, es posible esperar puntuaciones mayores en niveles de satisfacción y bienestar.

La arquitectura o contexto estimula, incentiva, encamina, censura o prohíbe la toma de decisiones en relación con el bienestar y desarrollo de las personas que conviven en este contexto (Sunstein, 2016, 2019; Thaler, 2018). Al respecto, este artículo indaga por lo siguiente: ¿Qué arquitectura para la toma de decisiones se promueve en los programas institucionales de bienestar y desarrollo humano ofrecidos en tres instituciones de educación superior de la ciudad de Medellín y el municipio de Bello en Antioquia, Colombia?

\section{Método}

\section{Tipo de Estudio}

La presente investigación es un estudio de casos múltiples, utilizando un enfoque hermenéutico en la comprensión del discurso de los participantes. Dicho estudio permite conocer en profundidad y detalle los departamentos de bienestar estudiantil de tres universidades, con poblaciones ubicadas en niveles socio-económicos diferentes. Lo anterior permite un estudio de las interacciones en un sistema delimitado con un abordaje holístico, sistemático y profundo (Lucca \& Berríos, 2003; Opie, 2004). El estudio de casos múltiples favorece un tratamiento de los datos en un sentido global y el entendimiento de interpretaciones en un determinado momento y contexto particular (Ander-Egg, 2003; Bogdan \& Biklen, 2003). Dicha delimitación contextual facilita la posterior triangulación de la información para la comprensión cabal del fenómeno de estudio. Al igual que otras formas de investigación cualitativa, el investigador es el instrumento principal en la recolección de datos y se realiza un análisis inductivo cuyos resultados son de tipo descriptivo (Merriam, 2002). El proceso, metódicamente planeado, se apoya en una recolección de información sistemática. Debido a que la intención es entender el caso en sí mismo, la selección de la muestra es intencionada, es decir, no es un muestra probabilística ni representativa. Esto ha sido denominado muestra basada en criterios o muestra con un propósito (Merriam, 2002; Mertens, 2005; NewtonSutter, 2006; Stake, 2006, 1996). Los criterios usados para la selección de los participantes fueron: responsables (arquitectos) de los programas institucionales de bienestar estudiantil y desarrollo humano, encargados de su diseño, ejecución y evaluación.

\section{Fuentes de Información}

Información documental de la investigación empírica en psicología sobre la interacción entre decisión, el bienestar y el desarrollo humano. Quince entrevistas semi-estructuradas a líderes encargados de los programas institucionales de bienestar y desarrollo humano en las universidades objeto de estudio. Posterior a las entrevistas, se decidió realizar un grupo focal (GF) con 10 estudiantes de la Universidad que reportó los niveles más bajos de asistencia a las actividades. Los encuentros respetaron los protocolos de consentimiento libre e informado. Tanto las entrevistas como el grupo focal se realizaron en un solo momento (abordaje transversal).

\section{Instrumentos de Recolección de Información}

Fichaje bibliográfico en bases de datos para la discusión de resultados; entrevistas semi-estructuradas basadas en categorías iniciales de análisis y guía de grupo focal.

\section{Análisis de la Información}

En la presente investigación se siguieron los procesos descritos por Hoyos (2000) en el análisis de datos cualitativos: (a)Preparatoria: En esta fase se rastrea y ubica el material pertinente para la investigación y se esbozan unas categorías iniciales que permitan agrupar y organizar la información. Dicho sistema categorial se basó inicialmente en las unidades de análisis de los objetivos específicos de la investigación, y gracias al diseño flexible, pudo ser modificado de acuerdo con el desarrollo de la investigación y en beneficio de la misma. (b)Descriptiva: En esta fase se afina el sistema categorial de acuerdo con el análisis de la información desarrollado a partir de las unidades de análisis (fuentes documentales y entrevistas). (c)Interpretativa: 
En esta etapa se procede mediante la labor hermenéutica propiamente dicha, en la que se procuran establecer conexiones y comparaciones al interior de las unidades de análisis y entre ellas; se problematizaron las categorías; se buscaron develar vacíos, incoherencias, lagunas y vacíos en el conocimiento. (d)Construcción teórica global: Consiste en la formalización escrita de los resultados obtenidos que se plasman en el informe final y este artículo de investigación.

\section{Resultados}

\section{Creación y Seguimiento de los Programas Institu- cionales}

Las primeras dos categorías que permiten presentar los resultados y dar respuesta a la pregunta de investigación son las siguientes: 1. Creación de los programas institucionales de bienestar y desarrollo humano: Hace referencia al diseño de programas y actividades en función de la población atendida, cuyo propósito es impactar de forma satisfactoria las necesidades identificadas. En esta las subcategorías son: población atendida; necesidades; programas de bienestar y desarrollo; y creación de los programas. 2. Seguimiento de los programas institucionales: Implica la evaluación de los programas y las estrategias que disponen para su implementación. Las subcategorías que la componen son: impacto, contexto y evaluación.

Creación de los programas de bienestar y desarrollo humano. En esta categoría se identificaron diferencias significativas en la población atendida en las tres instituciones universitarias, debido principalmente a las características socio-económicas de los estudiantes matriculados. También, una marcada tendencia a combinar estudio-trabajo en la población que pertenece a los estratos 1, 2 y 3 , lo cual dificulta la participación en las actividades que ofrece el departamento de bienestar. Entre las razones para sostener tal estilo de vida se incluyen: necesidad de trabajar para pagar sus respectivos estudios; sostener económica y afectivamente uno o más hijos, o apoyar la economía familiar. Así lo expresan dos entrevistados: "Yo trabajo de 2 a 10, entonces yo cojo materias por la mañana, [...] mire que por la tarde tampoco me da, entonces por eso sí es falta de tiempo" (GF, participante 15). "En la universidad se ofrecen muchos espacios para que la gente aproveche, pero en realidad es minoría quienes lo hacen, puesto que la mayoría trabajan y estudian en las noches" (Participante 4).

La población restante, sin dificultades asociadas a la disponibilidad de tiempo, puede agruparse en: aquellos que desconocen las actividades y programas; los que la conocen y no les interesa; o los que tienen la información, les interesa, pero no deciden participar. Lo anterior se evidencia cuando un estudiante afirma:

yo creo que es la falta de interés como propio [...] bueno sí o sea el tiempo es crucial claro está, pero si uno tiene claro y está motivado con su propia vida porque quiere un buen cuerpo, una buena salud, porque quiere aprender, tener más cosas para su hoja de vida, hace lo que sea por eso. (GF, participante 18)

Con diferencias en la población en cada universidad, la oferta de programas no satisface las mismas necesidades. Los encargados de tomar las decisiones reconocen que la creación de los programas está influenciada por las directrices administrativas, los discursos organizacionales, la tradición de la actividad per se (de tipo, "ya se venía ofreciendo hace muchos años en la institución"), iniciativas del equipo de trabajo, encuestas a los estudiantes, y sólo en dos ocasiones se basaron en investigaciones de campo con el propósito de identificar preferencias de los estudiantes. Esto se observa en algunos comentarios:

Nosotros diseñamos, no debería ser, pero diseñamos de acuerdo a lo que administrativamente creemos que es bueno para ellos, por ejemplo: vamos a darle a los muchachos guitarra, clases de guitarra. Nosotros no sabemos y no tenemos realmente cómo comprobar si a ellos les gusta la guitarra o realmente (lo que) les gusta es tocar la quena, pero les damos clases de guitarra y ellos entran a la clase de guitarra y ha sido una de las actividades más acogidas. (Participante 3 )

Sé que ahorita bienestar universitario está haciendo una revisión con una firma [...] buscando identificar los servicios que bienestar tiene y si los muchachos nos reconocen o no, los que nos reconocen ¿por qué?, los que no ¿por qué?, y ¿qué servicios creen que les hace falta de bienestar? (Participante 14)

Con respecto a las directrices administrativas se reconoce la subordinación del área frente a otras instancias institucionales. Aspectos asociados a la poca autonomía y la alta burocracia sobresalen en la mayoría de departamentos. Así lo ilustra un entrevistado:

Si es una toma de una decisión que depende de la alta dirección entonces se debe llevar qué es lo que necesitamos que se apruebe y por qué se necesita, cuál va ser el costo-beneficio, en qué me 
favorece como área de Bienestar, cuáles son los pro, cuáles son los contra. Lo que hace el rector, si él ve que es una decisión que puede tomarla dentro de su nivel jerárquico, aprueba la decisión que se va tomar, si no, entonces en un comité de rectoría o en un comité estatutario se toma esa decisión. (Participante 1)

Respecto al discurso organizacional, los entrevistados usan frecuentemente expresiones en torno al manejo del ocio y tiempo libre; formación de capacidades; acompañamiento psicológico; $y$ apoyo económico. Así se aprecia en las siguientes viñetas:

[trabajamos en el] fortalecimiento de competencias y de habilidades humanas que sean efectivas tanto para desenvolverse en un plano laboral como en un plano familiar o como en un plano social. (Participante 2)

Una palabra que nosotros usamos mucho es el deseo, [esto] es, digámosle, la chispa de vida que a vos te mueve en el mundo, y ahí es donde nosotros tratamos de vincularnos con eso, y que esa chispita se vuelva una llamita, para usar una metáfora; pero esa chispita, es diferente en cada quien, el trabajo fuerte de nosotros es ahí. Qué sí buscamos, que ellos logren ser la mejor versión de ellos. (Participante 13)

Hay una línea de cooperación y ayuda, entonces allí se enfoca todo el acompañamiento de los apoyos pretende responder a esas necesidades económicas que puedan presentar los estudiantes, acompañar frente a esas adversidades económicas, en cuanto a la alimentación, en cuanto al transporte y materiales. (Participante 7)

Cuando usan ocio y tiempo libre, se refieren a actividades como danza, teatro, música, deporte y cultura, incluyendo cine-foros. En relación con la formación de capacidades, se identificaron programas relacionados con afrontamiento del estrés, manejo de conflictos interpersonales y, consumo y abuso de sustancias psicoactivas. El componente económico busca impactar la permanencia estudiantil y con esto se incluye el soporte económico para matrícula, transporte, alimentación y materiales. Por último, a través de dispositivos clínicos se le ofrece al estudiante la posibilidad de discutir de manera individual su historia de vida.

Seguimiento de los programas institucionales. Hay consenso entre las tres instituciones en las dificultades que existen para medir el impacto de los programas institucionales. Con impacto, se refieren al efecto en la vida de las personas que participan de las actividades. Por tal motivo, deben acudir a otros recursos de medición asociados directamente con cobertura, frecuencia y satisfacción. Por cobertura se entiende número de estudiantes atendidos; en frecuencia se incluye el número de actividades/sesiones en las que participa; y en satisfacción, la percepción sobre la calidad del servicio recibido. En palabras de un entrevistado:

Realmente hablar de un impacto con evidencia, no lo tenemos. Nosotros vamos, siempre hacemos entrevistas, "a usted ¿qué le aportó, cómo le parece? En este momento nosotros solo estamos evaluando satisfacción. La calidad en términos de satisfacción. Con esta nueva forma de visionar el bienestar tenemos el reto de ser capaces de medir el impacto, porque hoy no tenemos evidencias. Tenemos evidencias en función de los testimonios, pero no de cifras. (Participante 6)

No se identificaron evaluaciones longitudinales de los servicios ofrecidos en bienestar, lo que indica la falta de trazabilidad de las actividades y el desconocimiento de su respectivo impacto a través del tiempo. Las instituciones para evaluar los programas poseen formatos físicos y digitales compuestos de preguntas cerradas con medición de escala, y preguntas abiertas para recoger observaciones y datos cualitativos de la experiencia. Con frecuencia estos formatos con preguntas generales no se adecúan a las actividades. En tanto provienen del área de certificación y calidad, el diseño de estos instrumentos no necesariamente incluye las necesidades del área de bienestar, y se usan a manera de plantillas de obligatorio diligenciamiento no ajustada a las situaciones singulares de cada intervención. En palabras de un participante:

La evaluación es una evaluación global genérica, se basa en varios aspectos y al final dice: aplica o no aplica. Nosotros creamos un formato y se formó un problema, porque inmediatamente nos dijeron: ustedes se salieron de lo estándar, de lo normal. [...] En la evaluación hay muchas cosas que son muy globales que no aplican. (Participante 4)

\section{Elementos de la Arquitectura para la Toma de Deci- siones}

Como tendencia general, no se evidencia un diseño para la toma de decisiones. Las instituciones con mayores recursos económicos realizaron investigaciones 
para conocer las preferencias de los estudiantes en cuanto a horarios de los servicios, mejores días para realizar las actividades y temas que evaluaban importantes. Dicha información fue considerada un recurso importante para tomar decisiones respecto a la creación, mantenimiento, modificación o eliminación de una actividad del área de bienestar. El cine-foro es un ejemplo de esto, se identificó mayor asistencia los viernes en lugar de los lunes, debido a que los estudiantes prefieren estas actividades más próximas al fin de semana. Asimismo, se observó que las decisiones importantes están centralizadas en los cargos de los jefes del departamento, pero en su ejecución se encontraron diferencias asociadas al estilo de dirección o la estructura organizacional que permitía mayor o menor flexibilidad y autonomía. Un participante lo explica en esta vía:

para la toma de decisiones, primero uno debe, como cabeza directamente de un programa, tener una cierta forma de libertad, porque es a veces un poco frustrante [...] que la decisión esté siempre, así sea muy mínima, en cabeza de la persona que está encima de nosotros. Ahí es complicado porque si vos tienes las cosas muy claras, si vos tenés una noción más amplia de tu área, vos podés perfectamente tomar una decisión, pero es incómodo cuando simplemente la decisión al compartirla no se pide consentimiento final. Eso genera un poco de frustración porque hay decisiones que uno perfectamente puede tomar, sin necesidad de ahondar, sin necesidad de escalar, pero desafortunadamente nosotros acá no lo podemos hacer, no lo hacemos, pues en última instancia es la decisión que se tome desde arriba. (Participante 4)

La planeación es concebida como una herramienta para evitar la incertidumbre, aunque los participantes reconocen que cada proceso humano tiene un componente que escapa al control y a la predicción. Un entrevistado lo explica de este modo:

Yo soy amante a la planeación, entonces una de mis tareas aquí en bienestar ha sido implementar métodos no tan rigurosos como yo quisiera, para poder cerrar la brecha de los imprevistos. Mientras más pueda yo prever qué puede pasar, puedo tener plan b, c, puedo tener alternativas; si ya el asunto me cogió así, definitivamente habrá que resolverlo de la mejor manera posible, pero no necesariamente es la más pertinente, o la más eficaz, o la que menos recursos consume, (Participante 6)

\section{Consideración sobre Categorías Emergentes}

Las categorías emergentes se obtuvieron a través de la revisión bibliográfica, conforme avanzaba la investigación, y las situaciones problemáticas que nombraron los participantes de manera persistente durante las entrevistas. En ellas sobresale, la ausencia de redes de trabajo entre universidades para compartir las estrategia efectivas y los factores críticos de éxito de prácticas implementadas. A pasar de pertenecer a una red de universidades (ASCUN), podría persistir la idea de concebir otros departamentos de bienestar estudiantil como competencia.

Por otra parte, el concepto bienestar tiene una extensa tradición filosófica e incluye otros conceptos que se solapan entre sí, como calidad de vida, felicidad o placer (Vázquez, 2009). Este aspecto merece una distinción para entender la agenda de trabajo de los departamentos asociados. Por una parte, se entiende bienestar como estado de satisfacción y ausencia de sufrimiento y, por la otra, como se nombra al departamento o área dentro de la organización. Al ser un concepto complejo, multicausal y multinivel, el alcance que el departamento de bienestar estudiantil tiene sobre la medida global es limitada, reduciéndola a pocas esferas de la vida humana. Dichas limitaciones se pueden circunscribir a la experiencia universitaria. Esta diferencia la subrayó un entrevistado cuando afirma:

No necesariamente idea o sensación de bienestar es lo mismo que bienestar como dependencia y como dirección. Los chicos pueden decir que lo que hace falta de bienestar es ¡más parqueaderos! Eso no es algo que la dirección de bienestar pueda satisfacer. (Participante 14)

En las entrevistas realizadas, hay un factor común con respecto a la dificultad de caracterizar la población. Reconocen que la mayoría de la información disponible fue recogida en la inscripción inicial. Es decir, no se cuenta con información actualizada sobre las necesidades cambiantes de la población. Algunos participantes la reconocen como una de las principales dificultades, ya que si no existe tal caracterización de los estudiantes y sus preferencias, no se podrá identificar si los servicios ofertados satisfacen sus necesidades.

Por otra parte, surgió como categoría emergente el esfuerzo en la comunicación de los programas. Las personas entrevistadas reconocen que una de las razones por lo que no asisten los estudiantes a las actividades es el desconocimiento de la oferta. Aunque en los departamentos de bienestar hacen uso de diferentes

Estudos de Psicologia, 24(3), julho a setembro de 2019, 247-257 
medios de comunicación (correos, piezas impresas por la universidad, afiches, redes sociales, notas en la radio, imágenes por los televisores), muchas actividades no cuentan con el aforo necesario.

Finalmente, hay un reconocimiento de que las actividades ofertadas más allá de ocupar el tiempo libre, tienen el propósito de desarrollar habilidades para la vida, asociadas incluso con la vocación:

Que el ¿para qué se va a meter en un grupo de danza? Yo le digo, no es solo un espacio para ocupar el tiempo libre, es un espacio para desarrollar capacidades. Eso permite también explorarte como ser humano. Más allá de ser estudiante, como persona qué te gusta, qué te mueve, qué te hace vibrar, y puede que ahí puedas encontrar que la danza es tu vida, o puedes simplemente desarrollar unas capacidades. (Participante 8)

\section{Discusión}

Los departamentos de bienestar institucional de las universidades estudiadas tienen en común en su misión el acompañamiento de los estudiantes en el desarrollo de sus capacidades: una vida saludable, el fomento de actitudes responsables, el disfrute de actividades del tiempo libre, y el apoyo económico y académico. Expresan un compromiso en las diversas dimensiones del ser humano: biológica, psicológica, social y espiritual. En su praxis cada departamento hace énfasis en una de estas dimensiones, en declaraciones como "universidad saludable", "construcción social", "responsabilidades compartidas", "desarrollo armónico del ser". Entienden al estudiante como un ser plural, con múltiples necesidades.

Guiados por las directrices institucionales y las actividades administrativas, no es frecuente la articulación en sus prácticas de la investigación empírica. Vincular investigaciones de vanguardia permitiría adecuar su oferta de programas a las necesidades cambiantes de la población atendida. Esta es la idea del Premio Nobel de Economía Richard Thaler y Cass Sunstein en Nudge, un pequeño empujón (Thaler \& Sunstain, 2009, 2011). Su propuesta de la arquitectura para la toma de decisiones, puede aplicarse en el diseño de programas que impacten el bienestar y el desarrollo humano de los estudiantes universitarios. Los "arquitectos" encargados de estos departamentos, con pequeños cambios en los contextos de decisión, incentivan, refuerzan, anulan o disminuyen tanto la construcción de capacidades como la participación de los estudiantes a los programas, con la precaución de respetar, fiel a este enfoque, su libertad de elección (Hansen, 2016; Sugden, 2009; Thaler \& Sunstein, 2009, 2011).

De lo anterior se pueden sustraer dos implicaciones para la arquitectura institucional. La primera, la necesidad de emplear técnicas y tecnologías para conocer cómo piensan, sienten y se comportan los estudiantes a través de una participación activa de los mismos. Resulta indispensable que el diseño, implementación y ejecución los incluya, de otra forma no son verdaderas oportunidades de elección. La segunda, resulta incoherente que universidades que, trabajan con el conocimiento, cuentan con grupos de investigación y tienen vinculados importantes académicos e investigadores, no hagan uso de su capacidad instalada para realizar investigaciones al interior de sus departamentos de bienestar. Es decir, no se conciben como clientes internos de su propio andamiaje institucional

Según y Thaler y Sunstein (2011) hay tres influencias sociales que pueden ser usadas como nudges en las instancias privadas o públicas, siendo ellas: la información, la presión de los demás y la impronta para causar buenos cambios a la gente. Los nudges deben ser creados por los arquitectos de las decisiones para intentar cambiar positivamente el "conservadurismo colectivo". El término fue usado por Richard H. Thaler para referir a la propensión de la gente de seguir una regla de un grupo preestablecida hace mucho tiempo, aunque sea perjudicial o que no sepan quién la creó, se seguirá perpetuando esta regla incluso cuando hay pruebas claras de que es nocivo para ellos.

Ahora bien, si dicha arquitectura inicia con un conocimiento de la población, la caracterización resultado de este proceso no puede estar limitada a las entrevistas de ingreso o las encuestas, en el mejor de los casos anuales, que realizan con los estudiantes. Para resolver esa situación, la economía conductual hace uso de laboratorios de inspiración (Inspiration Economy Labs) para identificar factores "ocultos" en el diseño de los programas y servicios. La idea de los laboratorios es poner a prueba las percepciones en el campo y aplicar los hallazgos en un diseño más efectivo (Buheji, 2018, 2017, 2016; Levitt \& List, 2009). La creación de laboratorios de bienestar les podría ayudar a identificar oportunidades ocultas, involucrar a las partes interesadas y dar un rol activo a los estudiantes para la solución de los problemas. Adicionalmente, en la actualidad hay herramientas tecnológicas que facilitan la adquisición de información, evitando los altos costos que tienen la contratación de empresas 
para la investigación aplicada. En este grupo de estrategias se incluye el diseño tecnologías basadas en la web y el uso de teléfonos inteligentes, la adquisición de software para medir bienestar y la denomina Social media research apoyados en el manejo de Big data (Subbu \& Vasilakos, 2017).

Una vez se obtiene esta información y se identifican las necesidades poblacionales, el siguiente paso es discutir las capacidades a construir y los programas a desarrollar y/o modificar. Martha Nussbaum propone en su enfoque diez capacidades centrales: vida; salud física; integridad física; sentidos, imaginación y pensamiento; emociones; razón práctica; afiliación; relación con otras especies; juego; y control sobre el propio entorno, sea político o material (Nussbaum, 2012). Estas capacidades coinciden con la perspectiva de los Derechos Humanos, los cuales propenden por una vida digna, prologada, sana y creativa (Cfr. PNUD), aspectos fundamentales en la consecución del bienestar global. Para la autora, las capacidades le pertenecen en primer y prioritario lugar a las personas, propugnando el principio según el cual "cada persona es un fin en sí misma" (Nussbaum, 2012, p. 55). Al comparar las prácticas de los departamentos de bienestar con estas capacidades centrales, permanecen algunas sin ser desarrolladas. En esta vía, se puede mencionar el acompañamiento al desarrollo emocional (manejo de la ira, violencia, estrés, miedo, ansiedad), la reflexión crítica sobre la planificación de la propia vida (autonomía, autogestión), el fortalecimiento de las habilidades de afiliación e interacción social (toda acción que vulnere la dignidad humana) y la educación moral, ética y política (vida en común, responsabilidades ciudadanas y toma de decisiones políticas). Un programa integral de bienestar tendería, en esencia, a evitar lo que la autora llama elección trágica: momentos en que las capacidades colisionan entre sí. Este es el caso de los estudiantes que abandonan la universidad cuando no se concilian las necesidades económicas del núcleo familiar y la necesidad de formación profesional.

Por su parte, de acuerdo a los tres componentes propuestos por Oishi (2010) que incluye el concepto de bienestar: afectivo, cognitivo y eudaimónico, no se encontró evidencias de mediciones de bienestar de los estudiantes, aunque en dos universidades mencionaron investigaciones (dos en total) para identificar las preferencias y necesidades de la población. Es cierto que los departamentos de bienestar institucional evalúan satisfacción, pero se hace en el contexto de la evaluación de las actividades realizadas. Esto propone un desafío importante si se desea seguir la trazabilidad del pilar conceptual del departamento.

Respecto a la concepción de bienestar como felicidad, ¿se pueden educar a los estudiantes para que sean felices? y ¿qué programas de formación se deben ofrecer para lograrlo? De acuerdo a las variables sugeridas por la psicología positiva, se identificaron programas que se enmarcan dentro de las actividades voluntarias, algunas amparadas en estudios previos de preferencias. Sin embargo, dichas actividades obedecen en su mayoría a la oferta, no a la demanda. Esto, unido a la poca actualización, baja especificidad y a la tradición organizacional que se desea mantener, contribuye a la escasa participación de la población. Como las actividades voluntarias requieren de la iniciativa estudiantil, el reconocimiento de sus propias necesidades es crucial para el éxito en su ejecución. Debido a la dificultad de responder a las demandas individuales, los departamentos podrían apoyarse en los grupos estudiantiles para la creación, diseño, difusión y consiguiente participación. Una de las universidades aplicó la arquitectura para la toma de decisiones en esta vía: asignó espacios de trabajo a los grupos estudiantiles contiguos al departamento de bienestar institucional, se diseñaron los cubículos para favorecer la comunicación y el intercambio de ideas, y les asignaron un presupuesto anual compartido. Esta arquitectura redundó en desarrollo de autonomía, comunicación efectiva, trabajo colaborativo, decisiones en conjunto y libertad en la gestión de sus recursos. Con esto también aliviaron el antiguo problema de competencia y rivalidad entre dichos grupos. Así, el departamento de bienestar deja de trabajar aisladamente si empodera a los estudiantes.

En este sentido, una formación en toma de decisiones reclama un ejercicio preliminar de deliberación individual y colectiva entre los "arquitectos" o líderes encargados de diseñar y ejecutar los programas de bienestar institucional y, luego, evaluar cómo pequeños cambios deliberados de contexto inciden de forma esperable en las decisiones de los estudiantes. El primer ejercicio requiere evaluar posibles conflictos de interés, prejuicios y garantías de transparencia en información que pueden incidir de forma desafortunada en el proceso de diseño y ejecución (Ariely, 2012; Sunstein, 2017; Thaler \& Sunstein, 2009). Para esto es indispensable evaluar el pensamiento automático, social y basado en modelos mentales de los líderes (Grupo Banco Mundial, 2015; Sunstein, 2017; Thaler \& Sunstein, 2009). Y el segundo momento de formación 
implica realizar trabajo de campo para conocer las preferencias de los estudiantes y evaluar de manera sistemática los cambios de contexto (Grüne-Yanoff, 2016; World Bank Group, 2015). En este caso la correspondencia entre elección y bienestar constituye el criterio de evaluación de los programas ofertados (Grüne-Yanoff \& Hertwig, 2016; Mills, 2015; Thaler \& Sunstein, 2009). Efectivamente una arquitectura que no incluya la participación de su sujeto/objeto de estudio asume el riesgo de convertirse en un sistema asistencialista, donde el estudiante es sólo un receptor de beneficios psicosociales y económicos, sin participar activamente en la configuración de sus opciones de elección como oportunidades para el bienestar y el desarrollo humano.

Asimismo, una formación en toma de decisiones debe desarrollar la capacidad de deliberar los problemas que enfrentan los estudiantes. Esto incluye examinar, reflexionar, discutir y debatir con su grupo de trabajo las necesidades bio-psico-sociales de la población atendida. De igual forma, la capacidad de comprender la universidad como un todo, no sólo un departamento de bienestar institucional. Lo que implica establecer redes de trabajo inter e intra organizacionales, con docentes, personal administrativo, grupos estudiantiles y personal de apoyo, para que las acciones en materia de bienestar sean transversales a la vida universitaria. Esta red de apoyo, adicionalmente, permite el aprovechamiento de experiencias exitosas y evita recaer en errores tan costosos en las vidas de las personas (por ejemplo el abandono estudiantil). También, una formación en toma de decisiones puede desarrollar la capacidad de preocuparse por la vida de otros, de imaginar lo que las acciones institucionales significan en cuanto a las oportunidades y experiencias de los estudiantes, ciudadanos en formación, para que ellos no sean vistos como actores pasivos de sus programas de bienestar. Como lo explica Nussbaum (2016, p. 20) "una buena educación puede llevar a los jóvenes a sentir genuina compasión por las necesidades de los demás, y puede conducir a verlos como personas con derechos iguales a los suyos". Una genuina preocupación que se trasmita en su educación en las artes liberales, donde los universitarios se preparen para una ciudadanía democrática decente, no para convertirse en máquinas útiles (Nussbaum, 2016).

Finalmente, hay cambios en las vidas que no están sujetas por el principio de adaptación, por lo que generan un gran impacto en la medida del bienestar. El más importante de ellos son las relaciones interpersonales, esto es, el número de relaciones que tiene una persona y la calidad de las mismas (Lyubomirsky, King, \& Diener, 2005; Lyubomisrky, Sheldon, \& Schkade, 2005; Seligman, 2002). Para Haidt (2006, p. 120) "Las buenas relaciones hacen a las personas felices, y las personas felices tienen más y mejores relaciones que las que tienen las personas infelices. [...] las personas nunca se adaptan al conflicto interpersonal". Acorde con lo anterior, cobra importancia el desarrollo de capacidades asociadas con la educación sentimental y el manejo del conflicto interpersonal. Los espacios clínicos y psicoterapéuticos ofrecidos en las universidades apoyan este aspecto, pero la posibilidad de cobertura poblacional es mínima y no todos los estudiantes desean ir al psicólogo para sostener un proceso psicoterapéutico. Aún permanece la negativa de algunos estudiantes de pedir ayuda en situaciones de crisis personal.

\section{Conclusión}

El presente estudio tuvo como objetivo explorar la arquitectura para la toma de decisiones que se promueve en los programas institucionales de bienestar y desarrollo humano, ofrecidos en tres instituciones de educación superior. A través de entrevistas, grupos focal, revisión documental, se conocieron los programas institucionales y se identificaron los componentes asociados con el proceso de toma de decisiones, el rol del arquitecto y las influencias contextuales. Estos ejes de intervención son complejos, dinámicos e individuales, por lo que su consecución propone retos cambiantes a los arquitectos encargados de diseñar los programas. Como resultado, se diseñan contextos de decisión según el caso a caso. Para esto, es preciso una formación en toma de decisiones de los arquitectos de estos programas, mayor conocimiento de la población atendida, recursos destinados a la investigación y mayor participación de los estudiantes en el diseño mismo de los programas. Los departamentos al integrarse a la comunidad universitaria y trabajar conjuntamente con los estudiantes y grupos estudiantiles, generan relaciones cercanas y atentas a las necesidades emergentes. Trabajar aisladamente los aleja de las nuevas ideas, socaba sus iniciativas y disminuye su cobertura.

La metodología elegida en la presente investigación no permite realizar generalizaciones ni aseverar el comportamiento de los arquitectos en otras universidades. Para tales fines, se sugieren ampliar los casos elegidos y realizar una contrastación detallada del proceso de toma de decisiones, creación, implementación y evaluación de los programas. Sin embargo, el 
estudio de casos múltiples y la comprensión hermenéutica del discurso contribuye a la identificación de la arquitectura organizacional de las instituciones participantes; permite detectar las estrategias a implementar, si se quiere aplicar la economía conductual, y subraya las principales limitaciones de participación e impacto cuando no se conocen las necesidades de la población atendida, aspectos a resolver para impactar positivamente el bienestar de los estudiantes. En futuros estudios se sugiere implementar diseños mixtos donde la información cualitativa a obtener pueda contrastarse con un diseño experimental que mida el tamaño de efecto sobre el bienestar y el desarrollo humano de los estudiantes, cuando se realizan pequeñas modificaciones en la arquitectura de elección institucional y se implementen estrategias de formación de autonomía.

\section{Referencias}

Alkire, S. (2002). Dimensions of human development. World Development, 30(2), 181-205. doi: 10.1016/S0305-750X(01)00109-7

Álvarez, J. (2012). Amartya Sen. La concepción vigorosa de la libertad. Claves de Razón Práctica, 219, 76-82. ISSN 1130-3689

Ander-Egg, E. (2003). Métodos y técnicas de investigación social IV técnicas para la recogida de datos e información. México: Grupo Editorial Lumen Humanitas.

Ariely, D. (2012). Por qué mentimos... en especial a nosotros mismos. Barcelona: Editorial Planeta S.A.

Armitage, D., Bene, C., Charles, A., Johnson, D., \& Allison, E. (2012). The interplay of well- being and resilience in applying a socialecological perspective. Ecology and Society, 17(4), 15-32. doi: 10.5751/ES-04940-170415

Asociación Colombiana de Universidades(2017). Reglamentación de la Red ASCUN - Bienestar Universitario. Acuerdo 09 del 14 de agosto de 2017. Recuperado de https://ascun.org.co/uploads/default/netwo rks/34e9079a5508cda35ad0160f03c720ba.pdf

Bogdan, R., \& Biklen, S. (2003). Qualitative research for education: an introduction to theories and methods. Nueva York: Allyn \& Bacon.

Buheji, M. (2016). Handbook of inspiration economy. Bookboon. ISBN: 978-87-403-1318-5.

Buheji, M. (2017) Understanding problem solving in inspiration labs. American Journal of Industrial and Business Management, 7, 771-784. doi: 10.4236/ajibm.2017.76055

Buheji, M. (2018). Re-inventing our lives, a handbook for socio-economic problem solving. UK: Author House.

Contecha, L. F. (2008). Los sentidos del bienestar universitario en Rudecolombia: más allá de un servicio assistencial (Tesis doctoral, Universidad del Cauca, Colômbia). Recuperado de http://repositorio. unicauca.edu.co:8080/xmlui/handle/123456789/1240

Contecha, L. F., \& Jaramillo, L.G. (2011). El Bienestar Universitario subordinado a una modernidad instrumental. Revista Udca Actualidad \& Divulgación Científica, 14(1), 101-109. Recuerado de http://www.scielo.org.co/scielo. php?script=sci_arttext\&pid=S0123-42262011000100013\&lng=en\&tlng=
Diener, E., Suh, E., Lucas, R., \& Smith, H. (1999). Subjective well-being: Three decades of progress. Psychological Bulletin, 125, 276-302. doi: 10.1037/0033-2909.125.2.276

Grüne-Yanoff, T. (2016). Why behavioural policy needs mechanistic evidence. Economics and Philosophy, 32(3), 463-483. doi: 10.1017/ S0266267115000425

Grüne-Yanoff, T., \& Hertwig, R. (2016). Nudge versus boost: How coherent are policy and theory? Minds and Machines, 26(1-2), 149183. doi: 10.1007/s11023-015-9367-9

Grupo Banco Mundial (2015). Informe sobre el desarrollo mundial: mente, sociedad y conducta. Washington, DC.

Haidt, J. (2006). La hipótesis de la felicidad. Barcelona: Editorial Gedisa.

Hansen, P. (2016). The definition of nudge and libertarian paternalism: Does the hand fit the glove? European Journal of Risk Regulation, 7(1), 18-20. doi: 10.1017/S1867299X00005468

Helliwell, J., Barrington-Leigh, C., Harris, A., \& Huang, H. (2010). International evidence on the social context of well-being. En $\mathrm{E}$ Diener, J. Helliwell, \& D. Kahneman (Orgs.), International differences in well-being (pp. 291-327). Oxford: Oxford University Press.

Hoyos, C. (2000). Un modelo para la investigación documental. Guía teórico práctica sobre construcción de estados de arte. Medellín: Señal Editorial.

International Association of Universities (2016). Higher education paving the way to sustainable development: A global perspective. Report of the 2016 IAU Global Survey on Higher Education and Research for Sustainable Development. París: IAU. Recuperado de https://iau-aiu. net/IMG/pdf/higher-education-paving-the-way-to-sd-iau-2017.pdf

Kahneman, D. (2012). Pensar rápido, pensar despacio. Barcelona: Random House Mondadori, S.A.

Kitayama, S., Markus, H. R., \& Kurokawa, M. (2000). Culture, emotion, and well-being: Good feelings in Japan and the United States. Cognition \& Emotion, 14, 93-124. doi: 10.1080/026999300379003

Levitt, S., \& List., J (2009). Field experiments in economics: The past, the present, and the future. European Economic Review, 53(1): 1-18. doi: 10.3386/w14356

Lucca, N., \& Berríos, R. (2003). Investigación cualitativa en Educación y Ciencias Sociales. San Juan: Publicaciones Puertorriqueñas.

Lyubomirsky, S., King, L., \& Diener, E. (2005). The benefits of frequent positive affect: Does happiness lead to success? Psychological Bulletin, 131(6), 803-855. doi: 10.1037/0033-2909.131.6.803

Lyubomirsky, S., Sheldon, K., \& Schkade, D. (2005). Pursuing happiness: The architecture of sustainable change. Review of General Psychology, 9(2), 111-131. doi: 10.1037/1089-2680.9.2.111

Merriam, S. (2002). Qualitative research in practice. San Francisco: Jossey Bass.

Mertens, D. (2005). Research and evaluation in education and psychology. Thousand Oaks, Ca: Sage Publications

Mills, C. (2015). The heteronomy of choice architecture. Review of Philosophy and Psychology, 6(3), 495-509. doi: 10.1007/s13164-015-0242-7

Montoya-Vásquez, D., Urrego-Velásquez, D., \& Páez-Zapata, E. (2014). Experiencia en la coordinación de programas de bienestar universitario: la tensión entre el asistencialismo y el desarrollo humano de los estudiantes. Revista Facultad Nacional de Salud Pública, 32(3), 355-363.

Newton-Sutter, W. (2006). Introduction to educational research. Thousand Oaks, Ca: Sage Publications.

Estudos de Psicologia, 24(3), julho a setembro de 2019, 247-257 
Nussbaum, M. (2012). Crear capacidades: propuesta para el desarrollo humano. Barcelona: Piadós.

Nussbaum, M. (2016). Educación para el lucro, educación para la libertad. Entornos, 28(2), 138-145. Recuperado de https://journalusco.edu. co/index.php/entornos/article/view/1243/2390

Oishi, S. (2010). Culture and well-being: Conceptual and methodological issues. En E. Diener, J. Helliwell, \& D. Kahneman (Orgs.), International differences in well-being (pp. 34-69). Oxford: Oxford University Press.

Opie, C. (2004). Doing educational research a guide to first-time researchers. Thousand Oaks, Ca: Sage Publications.

Saldarriaga, A. E. (2010). El sujeto activo: Antropología política en Amartya Sen. Eidos: Revista de Filosofía de la Universidad del Norte, 13, 54-75. Recuperado de http://www.scielo.org.co/scielo.php?script=sci_ arttext\&pid=S1692-88572010000200004\&Ing=en\&tlng=

Seligman, M. (2002). Authentic happiness. Nueva York: Free Press.

Sen, A. (1996). Capacidad y bienestar. En M. Nussbaum \& A. Sen (Orgs.), Calidad de vida (pp. 54-83). México: Fondo de Cultura Económica.

Stake, R. (1996). The art of case study. Thousand Oaks. Ca: Sage Publications.

Stake, R. (2006). Multiple case study analysis. Nueva York:The Guildford Press.

Subbu, K., \& Vasilakos, A. (2017). Big data for context aware computing perspectives and challenges. Big Data Research, 10, 33-43 doi: 10.1016/j. bdr.2017.10.002
Sugden, R. (2009). On nudging: A review of nudge: Improving decisions about health, wealth and happiness by Richard $\mathrm{H}$. Thaler and Cass R. Sunstein. International Journal of the Economics of Business, 16(3), 365-373. doi: 10.1080/13571510903227064

Sunstein, C. (2016). The ethics of influence: Government in the age of behavioral science. Cambridge University Press.

Sunstein, C. (2017). Paternalismo libertario. Barcelona: Herder Editorial, S.L.

Sunstein, C. (2019). On freedom. Princeton University Press.

Thaler, R. (2018). Todo lo que he aprendido con la psicología económica. Deusto.

Thaler, R., \& Sunstein, C. (2009). Nudge. Improving Decisions about Health, Wealth and Hapiness. New York: Penguim Books.

Thaler, R., \& Sunstein, C. (2011). Un pequeño empujón (nudge). Madrid: Taurus.

Valarezo, K., \& Túñez, J. (2014). Responsabilidad social universitaria. Apuntes para un modelo de RSU. Revista de comunicación, 13 84-117. Recuperado de https://revistadecomunicacion.com/pdf/2014/ Art084-117.pdf

Veenhoven, R. (2009). How universal is happiness? En E. Diener, D. Kahneman, \& J. Helliwell (Orgs.), International differences in well-being. Nueva York: Oxford University Press.

World Bank Group (2015). World development report: Mind, society and behavior. Washington, DC.

1.Versión original: "1. Affect, Happy now?, Happy in general?; 2. Cognitive, Satisfied now? Satisfied in general?; 3. Eudaimonic, Meaningful now? Meaningful in general?"

Isabel Cristina Lopera, Ph.D. (c) en Ciencias Sociales en la Universidad de Salamanca, Estudiante doctoral en la Universidad de Salamanca. Dirección para correspondencia: Calle Peñuelas de San Blas 11, Bajo A, Salamanca, España, 37007. Teléfono: 923-294400 Ext. 3516. Correo electrónico: ilopera@eafit.edu.co

Jonathan Echeverri Álvarez, Ph.D. (c) en Filosofía, Filosofía de la Ciencia en la Universidad de Antioquia, Profesor Asistente en la Universidad EAFIT. Correo electrónico: jechev39@eafit.edu.co 